\title{
RESEARCH
}

Open Access

\section{Paving the way for a better understanding of the pathophysiology of gait impairment in myotonic dystrophy: a pilot study focusing on muscle networks}

Antonino Naro ${ }^{1}$, Simona Portaro ${ }^{1}$, Demetrio Milardi ${ }^{1}$, Luana Billeri ${ }^{1}$, Antonino Leo ${ }^{1}$, David Militi ${ }^{2}$, Placido Bramanti ${ }^{1}$ and Rocco Salvatore Calabrò ${ }^{1 *}$ (iD

\begin{abstract}
Background: A proper rehabilitation program targeting gait is mandatory to maintain the quality of life of patients with Myotonic dystrophy type 1 (DM1). Assuming that gait and balance impairment simply depend on the degree of muscle weakness is potentially misleading. In fact, the involvement of the Central Nervous System (CNS) in DM1 pathophysiology calls into account the deterioration of muscle coordination in gait impairment. Our study aimed at demonstrating the presence and role of muscle connectivity deterioration in patients with DM1 by a CNS perspective by investigating signal synergies using a time-frequency spectral coherence and multivariate analyses on lower limb muscles while walking upright. Further, we sought at determining whether muscle networks were abnormal secondarily to the muscle impairment or primarily to CNS damage (as DM1 is a multi-system disorder also involving the CNS). In other words, muscle network deterioration may depend on a weakening in signal synergies (that express the neural drive to muscles deduced from surface electromyography data).

Methods: Such an innovative approach to estimate muscle networks and signal synergies was carried out in seven patients with DM1 and ten healthy controls (HC).

Results: Patients with DM1 showed a commingling of low and high frequencies among muscle at both within- and between-limbs level, a weak direct neural coupling concerning inter-limb coordination, a modest network segregation, high integrative network properties, and an impoverishment in the available signal synergies, as compared to HCs. These network abnormalities were independent from muscle weakness and myotonia.

Conclusions: Our results suggest that gait impairment in patients with DM1 depends also on a muscle network deterioration that is secondary to signal synergy deterioration (related to CNS impairment). This suggests that muscle network deterioration may be a primary trait of DM1 rather than a maladaptive mechanism to muscle degeneration. This information may be useful concerning the implementation of proper rehabilitative strategies in patients with DM1. It will be indeed necessary not only addressing muscle weakness but also gait-related muscle connectivity to improve functional ambulation in such patients.
\end{abstract}

Keywords: Signal synergies, Muscle networks, Myotonic dystrophy type 1, Gait, Time-frequency analysis, Multivariate analysis

\footnotetext{
* Correspondence: salbro77@tiscali.it

${ }^{1}$ IRCCS Centro Neurolesi Bonino Pulejo, via Palermo, SS 113, Ctr. Casazza,

98124 Messina, Italy

Full list of author information is available at the end of the article
}

(c) The Author(s). 2019 Open Access This article is distributed under the terms of the Creative Commons Attribution 4.0 International License (http://creativecommons.org/licenses/by/4.0/), which permits unrestricted use, distribution, and

reproduction in any medium, provided you give appropriate credit to the original author(s) and the source, provide a link to the Creative Commons license, and indicate if changes were made. The Creative Commons Public Domain Dedication waiver (http://creativecommons.org/publicdomain/zero/1.0/) applies to the data made available in this article, unless otherwise stated. 


\section{Background}

Myotonic dystrophy type 1 (DM1) is a multi-system disorder involving skeletal muscles and extra-muscular districts, characterized by myotonia, and muscle weakness and atrophy. Patients with DM1 also complain of symptoms belonging to Central Nervous System (CNS) (with minor cognitive deficits, sleep disorders, mild cortical atrophy and white matter abnormalities), endocrine system (e.g. diabetes), osteoarticular system (dysmorphisms), eyes (cataracts), and heart (conduction defects) [1].

DM1 is the most common adult form of muscular dystrophy, and is due to the expansion of CTG-repeat in the non-coding 3' UTR of the myotonic dystrophy protein kinase gene of $\geq 50$ times [1]. The range of CTG repeats determines disease severity, being classified into mild (E1, 51-149 repeats), classic (E2a; 150-450 repeats; and E2b, 451-1000 repeats), and Congenital form (E3, > 1000 repeats) $[2,3]$.

In the E2 form, patients with DM1 complain of distal and axial muscle weakness, as well as myotonia in both upper and lower limbs [1]. This consequently impairs gait and balance, with an augmented risk of falls, and a negative impact on functional capacities of daily living [4]. A proper rehabilitation program targeting gait and balance is indeed mandatory to procrastinate the use of the wheelchair, to maintain patient's quality of life, and to maximize patient's physical and psychosocial functions, even in the late stages of the disease [5-7]. However, only a few data are available on how a proper gait and balance rehabilitation program has to be implemented in patients with DM1.

Also, converging data on gait and balance pathophysiology in DM1 are still missing [5]. Up to date, gait pathophysiology of DM1 has been investigated in small cohorts of patients by using lower-limb segmental kinematics from infrared-emitting diodes, kinematics analysis coupled with ground reaction force measurements and wireless electromyography (EMG) [8-10]. Altogether, these studies suggest that gait and balance impairment may simply depend on the degree of lowerlimb distal muscle weakness (with a variable contribution of myotonia). The correlation between postural impairment and gait limitation and between myotonia severity and gait impairment remains instead partially unclear [8-10].

It is worthy to remember that gait and balance impairment in patients with DM1 are of particular nature, as they are unavoidably affected by CNS involvement [11]. This calls into account a potentially significant role of the deterioration of muscle coordination in gait and balance abnormalities in patients with DM1. Muscle synergy is defined as the temporal pattern of co-activations of different muscles recruited by a single neural command signal [12-
14]. Indeed, a single muscle can be part of multiple muscle networks, and a single network can activate various muscles [14]. The pathophysiological role of muscle connectivity deterioration in gait abnormalities in DM1 is suggested by the observation that some patients complain of significant alterations in gait and balance despite a mild weakness and myotonia severity [15]. The knowledge of muscle connectivity impairment in patients with DM1 might be important in order to understand: 1) motor behavior adaptations concerning balance and gait; and 2) why recovering abnormal muscle coordination through patient-tailored rehabilitation programs may be helpful to improve functional gait.

Given the particular nature of the motoric deterioration in DM1 (i.e. of both central and peripheral origin), our study was aimed at demonstrating muscle connectivity deterioration in these patients by a CNS perspective, namely signal synergies [16]. About that, we carried out a timefrequency spectral coherence analysis applied on surface EMG data recorded from eight lower limb muscles while walking upright to identify the muscles networks involved in ambulation [17]. This caveat is fundamental given that we aimed at investigating the neural drive to muscles deduced from EMG data, whereas classic muscle synergy studies typically focus on covariations in the temporal domain purely (rather than a time-frequency approach as in our study). More in detail, we used the graph theoretical analyses to quantify the characteristics of the network topology and statistically compare the muscle networks during walking, so to verify the degree of preservation of the patterns of widespread connectivity between muscles that needs to be coordinated to walk (as reflected by the neural drive to muscles deduced from EMG data). Indeed, muscle networks usually show synchronizations at multiple distinct frequency bands, which reflect the neural synchrony involved [17]. Thus, the absence of different muscle networks across distinct frequency bands would suggest the deterioration of CNS networking subtending muscle network organization in DM1 [17]. Whether the postural impairment has a biasing effect on gait limitation in patients with DM1 (regardless of muscle network deterioration) was investigated using an electronic baropodometry. Last, we sought at determining whether the neural drive to muscles was abnormal because of either muscle impairment (i.e., maladaptive muscular networking) or CNS damage (given that DM1 is a multi-system disorder also involving the CNS, and muscle coordination is of neural origin).

\section{Methods \\ Participants}

Seven patients with a clinical, genetic, and electrophysiological evidence of DM1 (six females and one male, mean age of $35 \pm 18$ years) were included in the study 
between January and October 2018. The inclusion criteria were as it follows: no clinical or laboratory evidence of other neurological disorders or diseases affecting the peripheral nervous system; no orthopedic illness potentially interfering with ambulation; no intake of drugs modifying muscles and nerve excitability. Clinicaldemographic characteristics are reported in Table 1. Ten normal individuals were enrolled as control group (HC) (four males and six females, mean age of $38 \pm 5$ years). The study was reviewed and approved by the Ethics Committee of our institute (ID: IRCCSME\#41/2017), and conducted according to the principles expressed in the Declaration of Helsinki. All subjects gave their written informed consent to study participation. Subjects' experimental flow is summarized in Fig. 1.

\section{Gait analysis}

Surface myoelectric signals were sampled at $1 \mathrm{kHz}$ and filtered at $5-400 \mathrm{~Hz}$ using the Smart Analyzer (Version 1.10.469.0; BTS, Milan, Italy) from rectus femoris (RF), biceps femoris (BF), tibialis anterior (TA), and gastrocnemius medialis $(\mathrm{G})$ of both lower limbs. After careful preparation of the skin, the bipolar adhesive surface electrodes were placed over the muscle belly in the direction of the muscle fibers according to the European recommendations for surface electromyography (SENIAM) $[18,19]$. An inter-electrode distance of at least $2 \mathrm{~cm}$ was used to minimize cross-talk between EMG signals [20]. Thus, signals were analyzed for root-meansquare (RMS) (a temporal parameter estimating muscle activation) to investigate lower-limb muscle activation during gait. The different phases of gait cycle were signaled by an accelerometer (G-Sensor) (BTS, Milan, Italy) fixed to the lumbar level by a Velcro strap. Gait data were recorded while the participant walked along a 10-meter walkway at their usual walking speed. Gait data were collected twice. Participants wore tight fitted clothing and were barefoot. The BTS software furnishes a measure called gait quality index (GQI). This is an overall gait performance score reflecting the quality of gait (https://www.btsbioengineering.com/products/freeemg-surface-emg-semg/), where normal gait is reflected by a 60:40\% stance:swing ratio (SSR) (i.e., ratio between stance from heel strike to toe-off, and swing phase duration from toe-off to heel strike), and a normal step cadence (about $1.9 \mathrm{~Hz}$ ) and gait cycle duration (GCD) (about $1.1 \mathrm{~s}$ from one right heel strike -namely, the initial contact- to the next one -namely, end of terminal swing). GQI values were averaged from the two runs and used for subsequent analyses. The higher the GQI was, the better the gait performance resulted.

\section{Stance analysis}

For the baropodometric evaluation, all participants were instructed to maintain an upright standing bipodalic position on a force plate of the electronic baropodometer (EPS R-1-KINETEC; RO + TEN, Arcore, Italy), with the feet wide apart at 30 degrees, both arms at their sides, and looking straight ahead to a 3-m distant achromatic target. Participants had to stand as still as possible for $2 \mathrm{~min}$. The task was repeated twice. We measured: 1) the ellipse sway area, which was calculated based on the $90 \%$ of oscillations of center of pressure (COP) amplitude along the main axes of the ellipse (anterior-posterior and medial-lateral); the ellipse sway area was also calculated with the eyes closed to compute the Romberg quotient. 2) The COP path length was defined as the real displacement of the center of pressure in reference to the plantar support, i.e., of the center of the distribution of the total force applied to the supporting surface). 3) The Romberg quotient (ratio of ellipse sway area with eyes closed/open). 4) The stabilometric index, which is automatically calculated by the device, reflects the distribution of the vertical forces on the support plane and the stability of, and the energy used by, the subject to precisely maintain postural control [21]. 5) The static index, which is also automatically calculated by the

Table 1 DM1 clinical-demographic characteristics

\begin{tabular}{|c|c|c|c|c|c|}
\hline gender, age & CTG expansion $^{a}$ & DM1 onset & Comorbidities & CRS & MSS \\
\hline$F, 49 y$ & E2 & $25 y$ & headache & 2 & 2 \\
\hline$F, 23 y$ & E2 & $16 y$ & nasal turbinate stenosis & 2 & 2 \\
\hline M, 19y & E2 & $17 y$ & - & 1 & 3 \\
\hline$F, 45 y$ & E2 & $22 y$ & BS1, OSAS & 2 & 3 \\
\hline$F, 29 y$ & E2 & $15 y$ & headache & 2 & 2 \\
\hline$F, 17 y$ & E2 & $15 y$ & - & 1 & 3 \\
\hline$F, 65 y$ & E2 & $30 y$ & headache, HBV-hepatitis, slight respiratory failure & 2 & 2 \\
\hline
\end{tabular}

Legend: CRS (Clinical rating scale) for DM1 (Dystrophic Myotonia type 1): $1=$ presence of myotonia and/or mild functional weakness without functional impairment; 2 =moderate muscle weakness leading to some degree of functional impairment; 3 = muscle weakness with severe functional impairment and in some cases resulting in the subjects being bound to a wheelchair; $4=$ bedridden. MSS (myotonia severity scale) for DM1 from 0 (absence of myotonia) to 4 (the worst myotonia experienced). BS1 Brugada syndrome type 1.OSAS Obstructive Sleep Apnea Syndrome. ${ }^{\text {a }}$ Range of CTG expansion: E1: 20-150; E2: 150-1000; E3: $>1000$ 


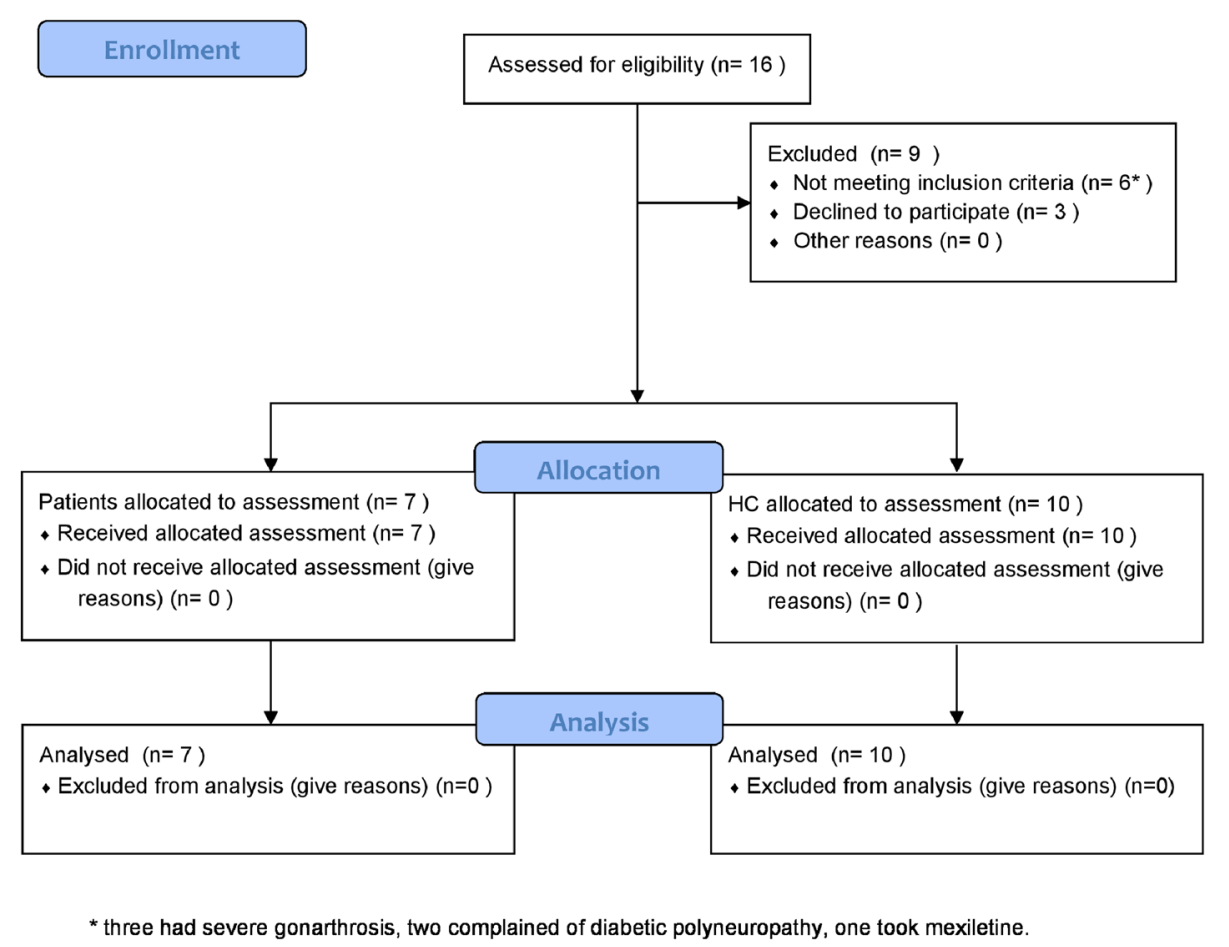

Fig. 1 Subjects' experimental flow

device, reflects the average position of the center of gravity of the body and its standard deviation [21].

\section{Muscle connectivity analysis}

EMG data processing preceding signal synergy extraction consisted of six sequential steps [17].

1) the EMG signals recorded from the eight surface EMG sensors were band-pass re filtered (0.5-70 $\mathrm{Hz}$ ), down-sampled to $200 \mathrm{~Hz}$, and rectified using Hilbert transform.

2) we extracted the power spectral density (PSD) (to investigate the spectral content of the EMG envelopes), which was individually normalized by the spectral resolution employed to digitize the signal, so to investigate the spectral content of the EMG envelopes ( $2 \mathrm{~Hz}$ bandwidth, thus obtaining 35 bins).

3) we estimated the intermuscular coherence (IMCoh) of the EMG envelopes to map undirected functional muscle networks (using Welch method with window length of $1 \mathrm{~s}$ and an overlap of $750 \mu \mathrm{s}$, averaging and squaring the values across the two trials to obtain magnitude-squared coherence) [22]. Coherence is commonly used to investigate the linear coupling between neural activities [23]. Specifically, the IMCoh estimated the descending output to different muscle groups between all pairs of muscles tested during walking at self-paced speed, so to define the edges of the undirected muscle network [24].

4) we calculated the partial directed coherence (PDC) (a measure of network connectivity) to map the directed muscle networks accounting for the temporal flow of information among muscles. To this end, we used the coefficients of a multivariate autoregressive model [25]. We had to estimate both undirected and directed graph as they reflect different network properties. The former graph (undirected network) considers a set of objects (vertices or nodes or points or actors) that are connected together with bidirectional edges (links or lines or ties). The latter (directed network or diagraphs) consider nodes where the edges (arcs) point in a direction. Thus, the undirected network reflects the functional associations between nodes (namely, muscles), whereas the directed network refers to the node-wise direction of information flow $[26,27]$. We also had to calculate PDC because IMCoh could be biased by common inputs arising from other structures (indirect paths), thus overestimating the strength of direct connections between motor-unit pools innervating different muscles [28]. To do that, we individually defined the optimal model order using Akaike's information criterion. Then, we computed the magnitude-squared Coh from the model coefficients 
to compare the model with the Coh spectra directly estimated from the data, in order to validate the model.

5) the network topology was studied using both of the undirected (IMCoh data) and directed (PDC data) muscle network connectivity measures in distinct frequency components (and their corresponding coupling strength by applying a non-negative matrix factorization -NMF). Frequency decomposition into non-negative factors was obtained using an alternating least squares algorithm applied to $2 \mathrm{~Hz}$ bins. Thus, we obtained two nonnegative matrices for each frequency bin reflecting one the pair-wise muscle spectral signatures (basis vectors), the other the pair-wise muscle connectivity strength.

6) these last data were used to calculate the network measures clustering coefficient $(\mathrm{CC})$, global efficiency (GE), and betweenness-centrality (BC). The $\mathrm{CC}$ is a measure of functional segregation and is equivalent to the fraction of the node's neighbors that are also neighbors of each other [29]. The mean CC for the network reflects, on average, the prevalence of clustered connectivity around individual nodes [30], with higher values indicating a more functionally segregated network. The average shortest path length between all pairs of nodes in the network is known as the characteristic path length of the network, which is the most commonly used measure of functional integration [29]. The average inverse shortest path length is a related measure known as the GE. Higher values of GE indicate a more functionally integrated network. Paths length can be generalized for directed and weighted networks: weighted path length is equal to the total sum of individual link lengths, which are inversely related to edge weights [30]. BC is a measure of centrality used to identify hubs in a network. It is defined as the fraction of all shortest paths in the network, which pass through a given node. $\mathrm{BC}$ is computed equivalently on weighted and directed networks, if path lengths are computed on respective weighted or directed paths.

\section{Signals synergy analysis}

Classically, synergies can be deduced from EMG data using factor analyses like NMF or principal component analysis [31-34]. We estimated synergies from EMG data using an NMF method based on the Lee-Seung algorithm [33], which is a basic and fast NMF algorithm with multiplicative updating rules.

EMG data from each trial (gait cycle) were used as input to the NMF algorithm. Muscle activity signals $M$ were arranged to form an $m \times n$ matrix, where $m$ is the number of measured muscles and $n$ the number of samples. Thus, $\mathrm{M}_{m \times n}$ data was subjected to NMF algorithm (i.e., they were factorized using the Lee-Seung algorithm) to obtain the synergy matrix $\mathrm{W}$ and the synergy recruitment matrix $C$ according to the formula $M_{m \times}$ ${ }_{n}=\left(\mathrm{W}_{m k} \times \mathrm{C}_{n k}\right)+\mathrm{E}_{m \times n}$, where $k$ is the number of synergies and $\mathrm{E}$ the residuals. The number of synergies needed to reconstruct the original muscle activity data during gait was calculated on the variability accounted for (VAF) the values of $\mathrm{M}_{m \times n}$ and $\mathrm{E}_{m \times n}$. When VAF was higher than the $90 \%$ threshold, then the number of synergies was deemed sufficient to regard the factorization results $\mathrm{W}_{m k}$ and $\mathrm{C}_{n k}$ as representative of the data set $\mathrm{M}_{m \times n}$.

\section{Statistical analysis}

Descriptive statistics were presented for all outcomes for both groups. Kolmogorov-Smirnov test estimated the normality of the distribution of data (all data were normally distributed, $p>0.1$ ). One-way analysis of variance (ANOVA) (factor group: two levels) was used to compare static and gait parameter between patients with DM1 and HC with post-hoc comparison between groups (Bonferroni correction for multiple comparisons).

When comparing muscle connectivity data, ANOVA was added with the factors muscle pair (56 levels) and frequency bin (35 levels) were implemented in the ANOVA, beyond the factor group. Significance was set at $p<0.05$ for all tests. Bonferroni correction for multiple comparison was applied in post-hoc $t$-tests.

Pairwise correlations between all tested variables were assessed using Spearman's rank correlation coefficient. A multivariate regression analysis was used to determine whether and which factor(s) among muscle connectivity data influenced signal synergy. The statistical analyses were conducted using the Stat-View ${ }^{\circ}$ software program (Hulinks Inc.; Tokyo, Japan).

\section{Results}

\section{Stance and gait data}

Gait was abnormal in all patients concerning the measures we evaluated. Patients showed a slightly longer gait cycle duration, as compared to $\mathrm{HC}(1.4 \pm 0.7$ vs. $1.1 \pm$ $\left.0.9 \mathrm{~s} ; \mathrm{F}_{(1,15)}=4, p=0.04 \eta^{2}=0.2\right)$, lower GQI $(81 \pm 5$ vs. $\left.98 \pm 2 \% ; \mathrm{F}_{(1,15)}=8.1, p=0.009, \eta^{2}=0.35\right)$, and lower step cadence $\left(1.4 \pm 0.7\right.$ vs. $1.9 \pm 0.1 \mathrm{~Hz} ; \mathrm{F}_{(1,15)}=113, p<0.001$, $\left.\eta^{2}=0.88\right)$, whereas there were no group differences concerning SSR $\left(1.5 \pm 0.5\right.$ vs. $1.6 \pm 0.5 ; \mathrm{F}_{(1,15)}=3, p=$ $\left.0.09, \eta^{2}=0.16\right)$. Further, patients walked at a slower pace than $\mathrm{HC}$ did $\left(0.6 \pm 0.04\right.$ vs. $1.2 \pm 0.01 \mathrm{~m} / \mathrm{s} ; \mathrm{F}_{(1,15)}=72$, $\left.p<0.001, \eta^{2}=0.83\right)$.

Balance presented clear abnormalities in patients with DM1, as compared to HC. Patients showed greater COP path length (556 \pm 40 vs. $370 \pm 57 \mathrm{~mm} ; \mathrm{F}_{(1,15)}=102, p<$ $\left.0.001, \eta^{2}=0.87\right)$, ellipse sway area $(163 \pm 30$ vs. $125 \pm 39$ $\left.\mathrm{mm}^{2} ; \mathrm{F}_{(1,15)}=24, p=0.001, \eta^{2}=0.57\right)$, stabilometric 
index $\left(21 \pm 3.5\right.$ vs. $8 \pm 1.5$; $F_{(1,15)}=17, p=0.002, \eta^{2}=$ $0.48)$, and static index $\left(9 \pm 1.2\right.$ vs. $5 \pm 1.5 ; F_{(1,15)}=19$, $\left.\mathrm{p}=0.001, \eta^{2}=0.55\right)$. There were no group differences concerning Romberg quotient $(0.9 \pm 0.2$ vs. $1.1 \pm 0.2$, $p=0.2$ ).

\section{Muscle connectivity data}

Both the groups showed similar PSD values across muscles (group $\times$ muscle interaction $p=0.9$ ), but there was a significant difference concerning frequency bins. In fact, patients showed a small frequency peak around $6 \mathrm{~Hz}$, whereas $\mathrm{HC}$ peaked around $10 \mathrm{~Hz}$ (group $\times$ frequency interaction $\mathrm{F}_{(34,510)}=64, p<0.001, \eta^{2}=0.65$ ) (Fig. 2).

The groups showed different IMCoh values across muscle pairs and frequency bins (group $\times$ muscle-pair $\times$ frequency-bin main interaction $\mathrm{F}_{(1870,28050)}=14, \mathrm{p}<0.001$, $\eta^{2}=0.48$ ). In particular, four main frequency components emerged after the decomposition of the EMG frequency spectra (Fig. 3). A component peaked around $5 \mathrm{~Hz}$ and indicated high Coh between proximal and distal of both sides. The second $(5-12 \mathrm{~Hz})$, third $(12-$ $25 \mathrm{~Hz})$, and fourth component $(25-45 \mathrm{~Hz})$ substantially involved the same muscle pairs. Thus, we found a gradual transition from a high bilateral IMCoh between lower leg muscles for the lowest frequency component observed in $\mathrm{HC}$ to a strong IMCoh with upper-lower leg muscles for the highest frequency components, in parallel to a reduced inter-limb IMCoh (Fig. 3). In particular, IMCoh in patients was stronger for proximal-distal muscle pairs within the same leg, and proximal-proximal and distaldistal muscle pairs of both limbs (RF_right-S_left $p=0.02$, RF_right-S_right $p=0.01, \mathrm{RF}$ _right-TA_left $\mathrm{p}=0.01$, and RF_right-TA_right $\mathrm{p}=0.01)$. On the contrary, IMCoh was high only concerning RF-S and TA-S of both lower limbs, both TA and both RF (all $p<0.001$ ). HC showed instead a strong IMCoh limitedly to TA-S of both limbs

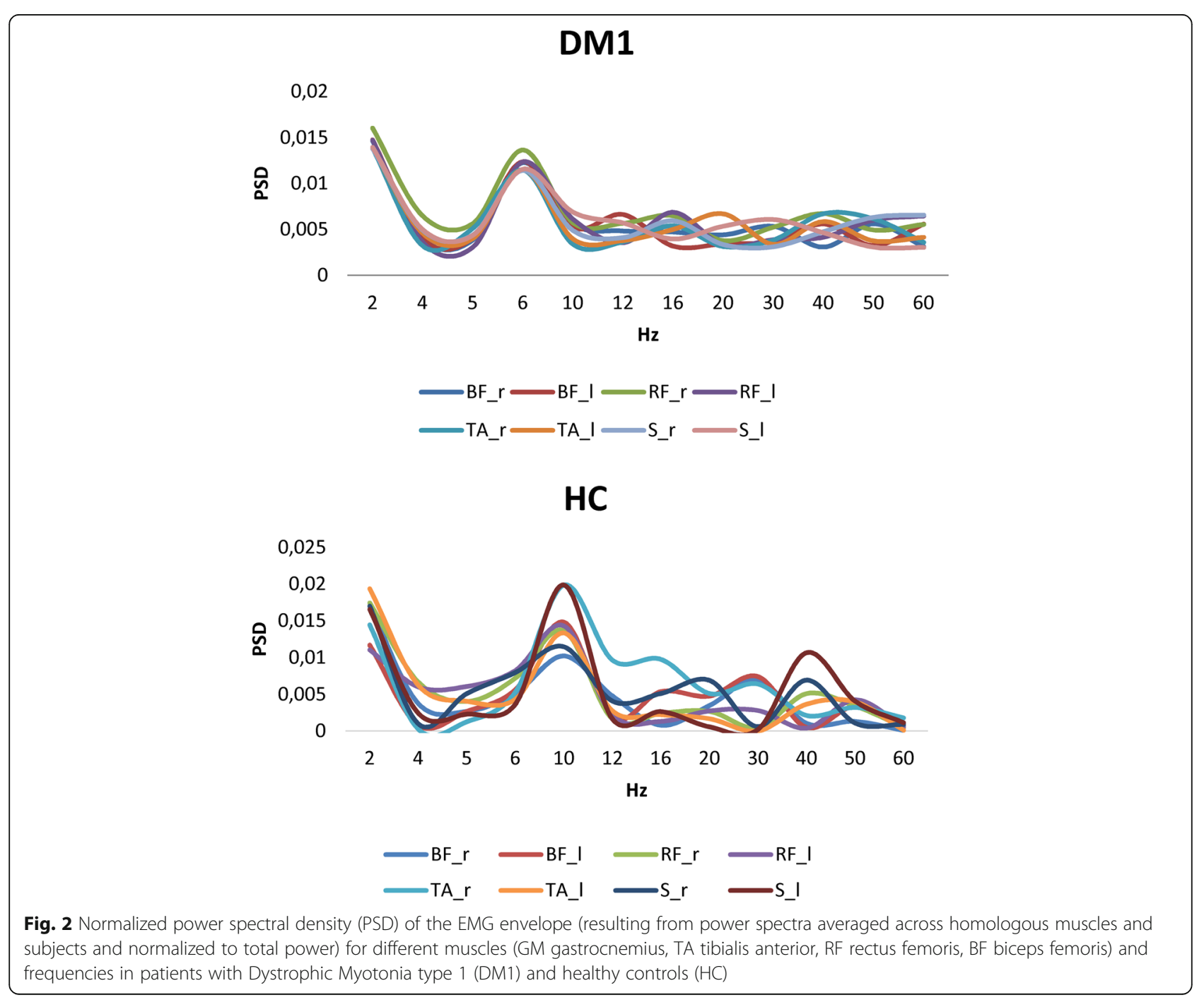




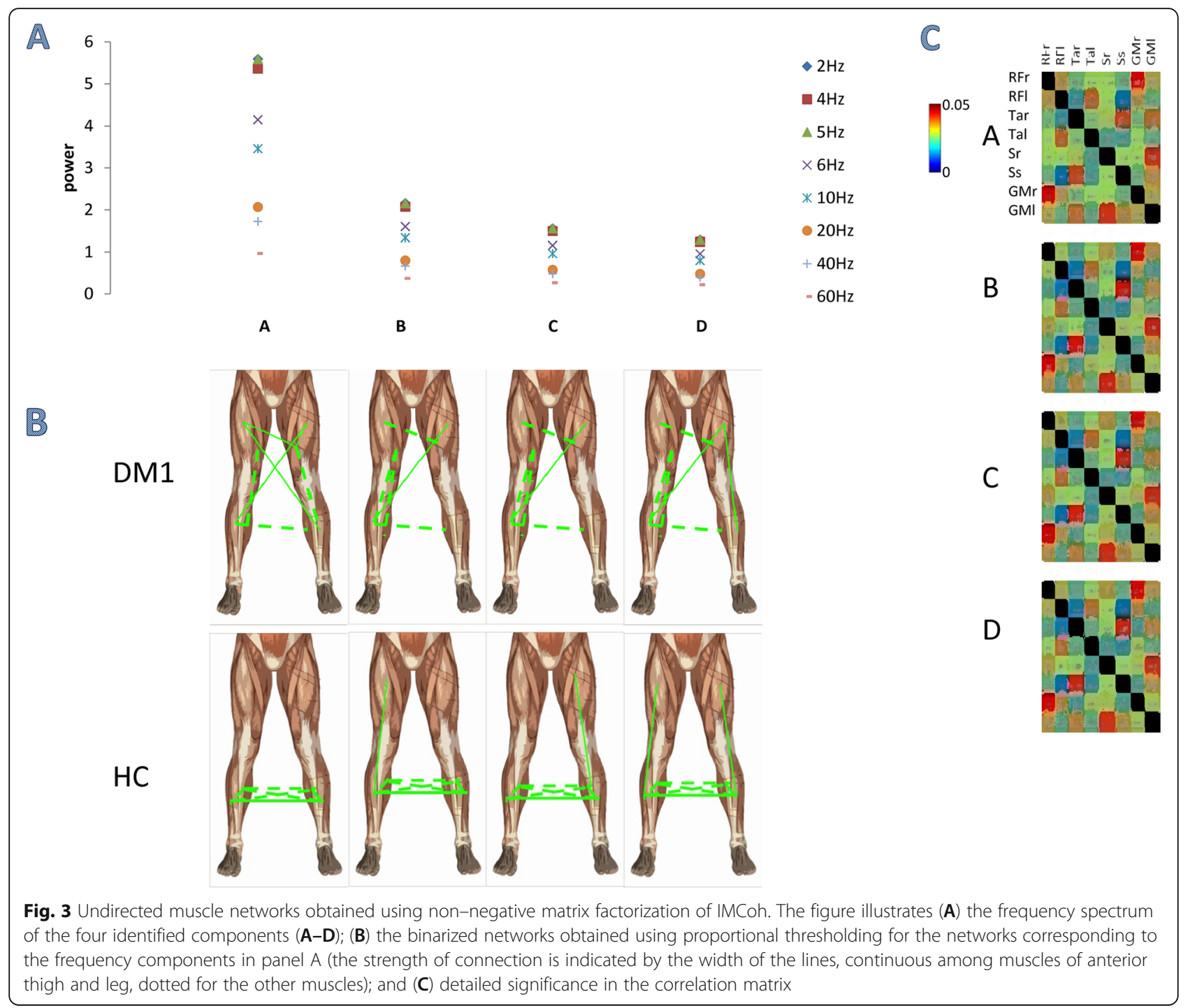

and VL-TA in each side. Each between-group comparison for each muscle pair and component was significant $(p<0.001)$.

The groups showed different PDC values across muscle pairs and frequency bins (group $\times$ muscle-pair $\times-$ frequency-bin main interaction $\mathrm{F}_{(1870,28050)}=9, \quad p<$ $0.001, \eta^{2}=0.37$ ). We identified four main directed components (Fig. 4) that were wider (i.e., involved more muscle pairs) than the undirected components (Fig. 3). This suggests the presence of different, strong pairwise connections in each of the components. Overall, PDC had lower values than IMCoh. This depends on the fact that PDC is less affected by inputs from other structures. HC showed instead a directed muscle network pattern characterized by high connectivity between the distal muscles within and between legs (Fig. 4). Each between-group comparison was significant for each muscle pair and component $(p<0.001)$.
Complex network analysis showed a trend to network integration (as reflected by $\mathrm{GE}$ and $\mathrm{BC}$ values) to the detriment of network segregation (as reflected by $\mathrm{CC}$ values) (Fig. 5). In detail, the CC was lower than $\mathrm{BC}(p<0.001)$ and $\operatorname{GE}(p<0.001)$, with particular regard to the first than the other components in the undirected network $(p<0.001)$ (Fig. 5). Within GE, the last component was higher than the other ones $(p=$ 0.01 ) (Fig. 5). This pattern was totally reversed in the $\mathrm{HC}$, i.e., a higher CC than $\mathrm{BC}(\mathrm{p}<0.001)$ and $\mathrm{GE}(p<$ $0.001)$. Within each graph measure, the last component was greater than the others in the undirected network $(p<0.001)$, whereas both BC and GE showed non-significant differences. For the directed networks, the trend of the network measures was the same observed in the undirected networks, but there were no significant pair-wise differences between the components in both DM1 and HC group (Fig. 5). 


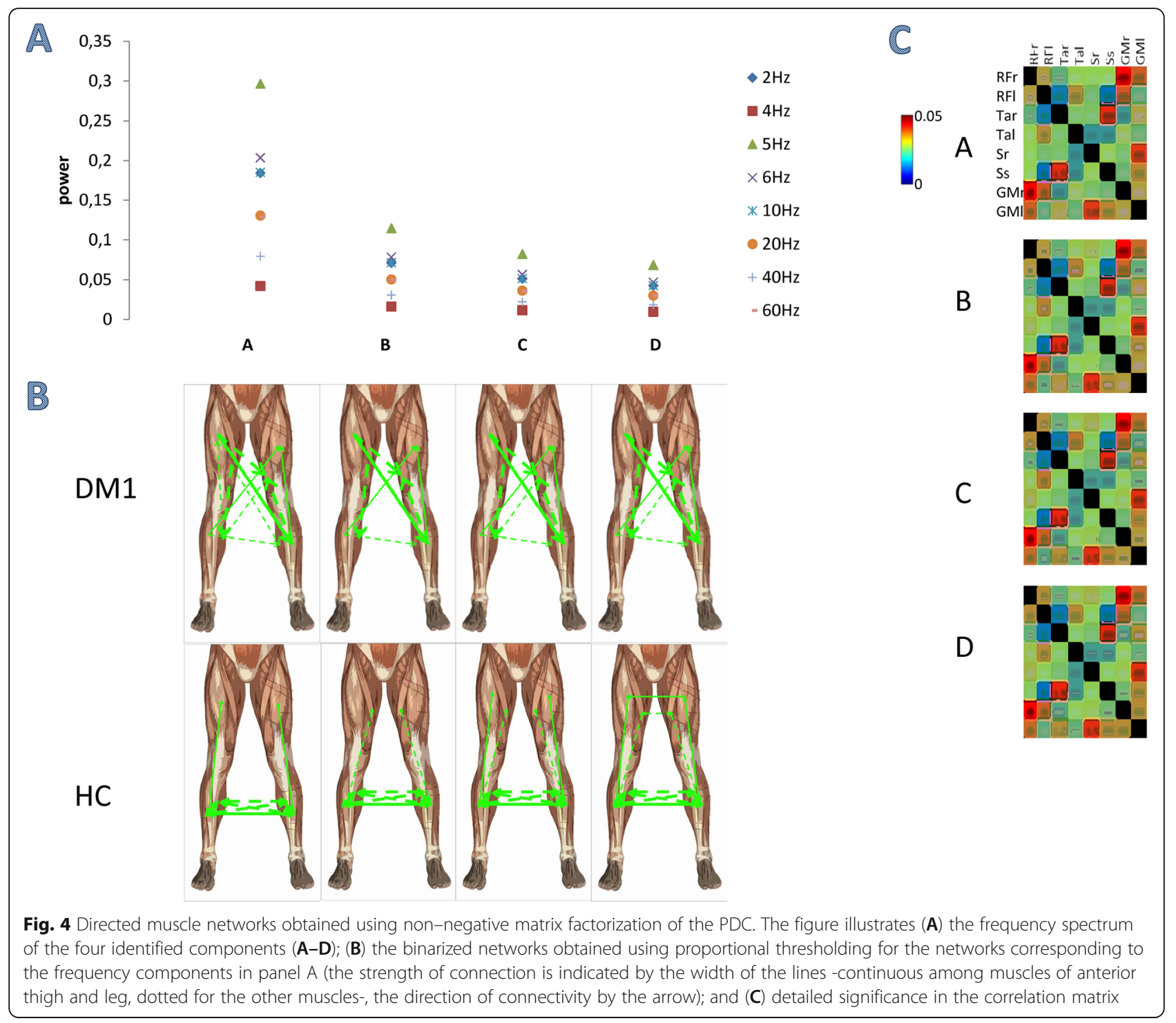

\section{Signal synergies data}

VAF calculation indicated that one to two synergies $k$ were needed to reconstruct the original muscle activity data during gait of all measured muscles in HCs. Thus, we extracted two synergies across participants to facilitate comparison (Fig. 6). Conversely, five to six synergies were required in patients with DM1 (Fig. 7). Patients with DM1 showed that muscle activation varied from a gait cycle to another as compared to HCs (higher $C$ values, $p<0.001$ ) (Figs. 6 and 7 ). In addition, $\mathrm{HC}$ used the same synergies across gait cycles (involving $\mathrm{S}$ and $\mathrm{TA}$ ), whereas patients used different synergies from a gait cycle to another (involving $\mathrm{BF}_{-}$ right, RF_right and TA_left or RF_left, both TA, and both $\mathrm{S})$, as reflected by the higher $\mathrm{W}$ values in $\mathrm{HCs}$ $(p<0.001)$ (Figs. 6 and 7).

\section{Pair-wise correlations}

Only the most significant gait and balance features were subjected to correlation analysis with respect to muscle connectivity and signal synergy data. Spearman's rank correlation test indicated a significant correlation between muscle weakness and stability index $(\mathrm{r}=-0.724, p=$ $0.002)$ and GQI $(r=-0.621, p=0.01)$. No correlations were found between muscle weakness and any of the muscle connectivity and synergy data. Among these, overall PDC values across muscle pairs and frequency bins significantly affected the overall synergy variance during gait $\left(\mathrm{R}^{2}=0.895, \mathrm{~F}_{(1870,28050)}=43, p<0.001, \eta^{2}=0.74\right)$.

\section{Discussion}

Muscle weakness clearly affects gait in patients with neuromuscular disorders of non-neural origin (e.g., 


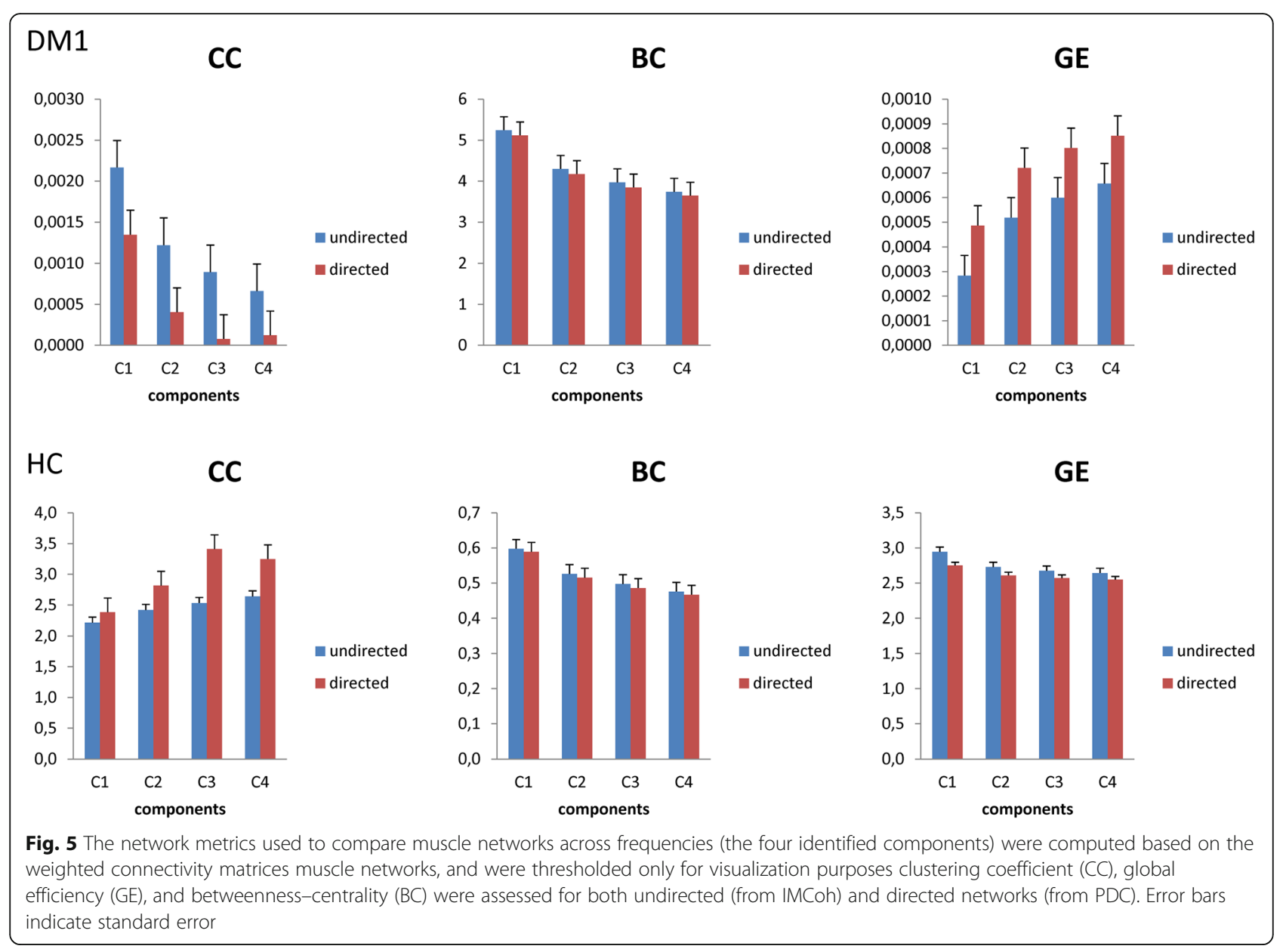

muscular dystrophy) as suggested by previous studies on gait analysis in patients with myopathy $[8,9,35,36]$. As expected, both balance and gait features were largely abnormal in patients and were significantly related to muscle weakness [37].

Whether muscle weakness directly contributes to muscle network deterioration or muscle network deterioration simply reflects neural impairments remains instead to be clarified. A previous study found that muscle weakness and muscle coordination are not correlated in muscle dystrophy [38]. However, gait and balance impairment in patients with DM1 may have peculiar aspects owing to the involvement of the CNS [39-41], thus potentially biasing muscle coordination. To this end, we employed a time-frequency approach on EMG data analysis rather than focusing on covariations in the temporal domain, so to gain information on signal rather than muscle synergies. To the best of our knowledge, this is the first attempt to study the CNS networking subtending muscle networks during walking upright in patients with DM1.

Muscle connectivity analysis offered three new data concerning a causative correlation between gait and balance impairment and the deterioration of muscle networking (i.e., beyond muscle weakness on its own).

1) Patients with DM1 show a deterioration of motor network organization as suggested by the IMCoh and PDC patterns. The former reflects muscle indirect connectivity (i.e., the statistical association between muscle activities), the latter the direct connectivity (i.e., the direction and the temporal flow of information within muscle pairs) [16, 42]. In particular, undirected connectivity maps revealed that patients complained of a commingling of low and high frequency ranges within both short- and long-range connections. In other words, functional muscle connectivity was observed at multiple distinct frequencies and, conversely, multiple connections between different muscle pairs were observed at each frequency. Hence, patients missed the functional connectivity patterns that occur normally to simplify and make more efficient integration processes (i.e., low frequencies within long-range connections and high frequencies within short-range connections). This denotes a 


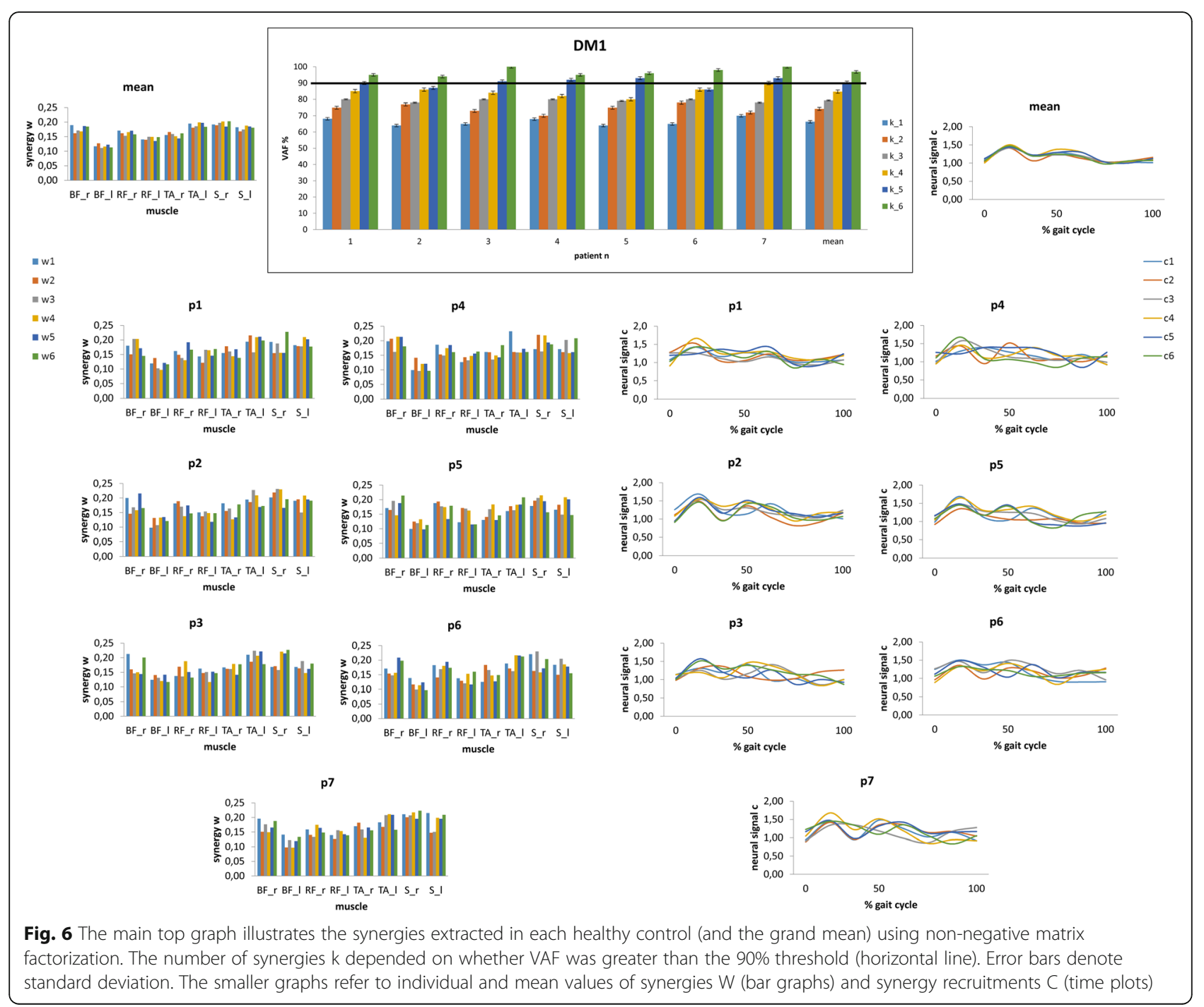

detrimental signal integration at different temporal and spatial scales, which could mirror what would occur at cortical network level owing to CNS involvement in DM1 [23, 43]. About that, it has been shown that peculiar patterns of fronto-parietal and cerebellum-cerebrum disconnection are detectable in patients with DM1, despite a global preservation of the brain topological properties [11, 44-46]. The evaluation of undirected connectivity maps was carried out by using PDC as a measure of network connectivity taking into account only the common input from other areas (thus being a more direct reflection of coupling between two areas) [25]. It revealed a loss of information flow directionality, i.e., there were multiple, bidirectional connections within and between muscle at both within- and between-leg level in patients with DM1. This directed network pathology may reflect a loss of the topological hierarchy at the level of M1 and, probably,
premotor-M1 connectivity, which are both important for muscle coordination [47, 48]. Noteworthy, no correlation existed between muscle weakness and muscle connectivity (both directed and undirected) deterioration.

2) Complex network analysis revealed a greater network integration to the detriment of network segregation, thus suggesting a reduced capacity of DM1 brain to segregate information into distinct modules. This may result in a signal synergy deterioration [47-49]. In fact, such a network deterioration suggests the presence of disorganized top-down projections from premotor to motor cortical networks with, consequently, a few common synaptic output to spinal networks (or central pattern generators) [50-53]. The distortion in top-down projections is also suggested by the recently reported abnormalities in corticospinal excitability and sensorimotor plasticity [54-58]. 


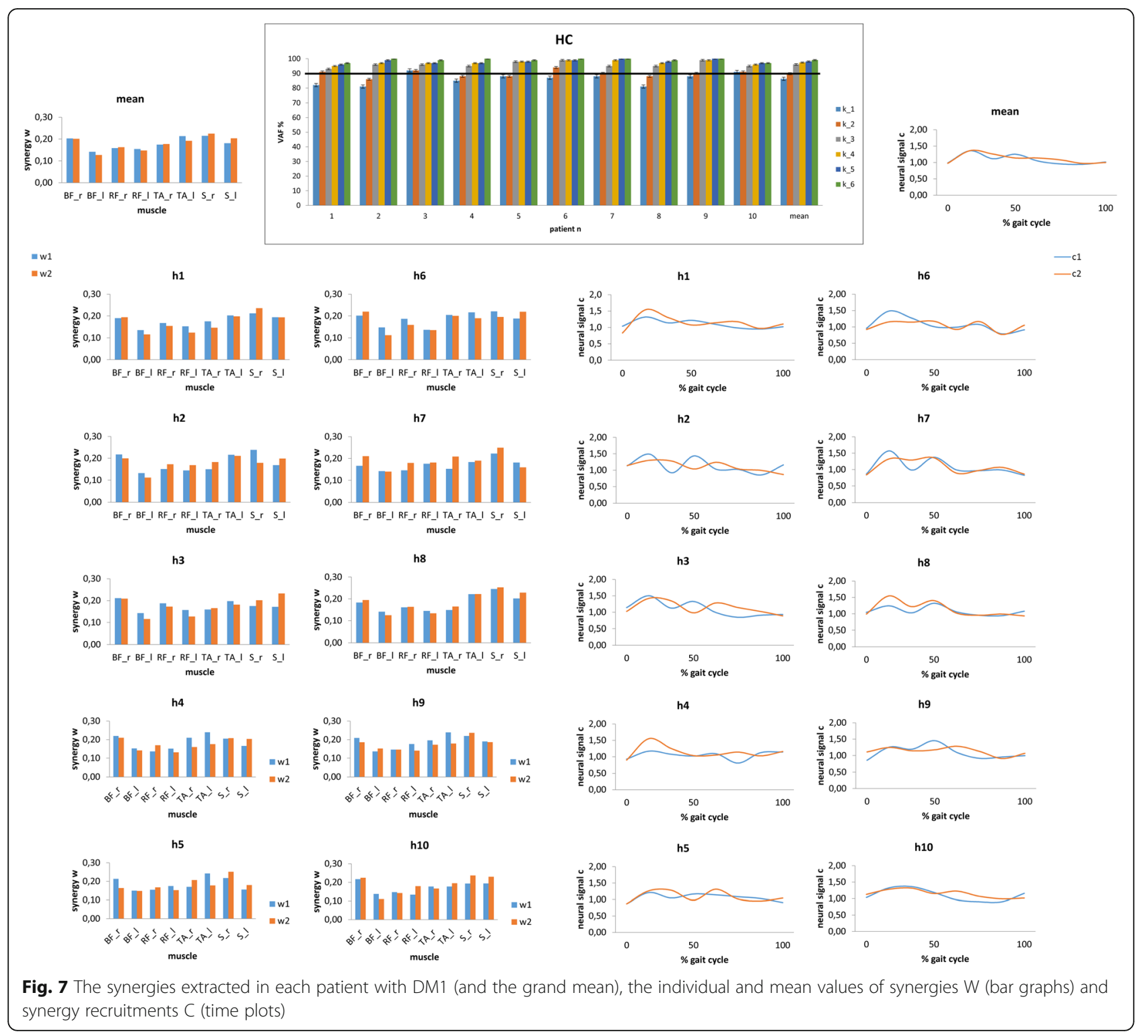

3) Patients showed an impoverishment in motor repertoire in terms of available synergies, i.e., more synergies were required to walk in patients as compared to HCs. In addition, a greater gait cycle to gait cycle synergy variability and a more variable number of synergies to be employed as well as of muscle to be activated were appreciable in patients with DM1, as compared to HCs. These data suggest that patients with DM1 suffered from a decreased complexity of motor control during gait (as pointed out by the high total variance accounted for by one synergy and by complex-network data analysis).

Moreover, the variability in the muscle required to walk indicated that the ankle joint strategy (as reflected by $\mathrm{S}$ and TA involvement) was sufficient to gait in HCs, whereas patients with DM1 had to harness both hip and ankle joint strategies in gait to compensate for synergy variability across gait cycles. Notably, no correlation existed between muscle weakness and muscular networking deterioration.

Altogether, these three findings suggest that gait and balance impairment in patients with DM1 may depend on the deterioration of the neuromuscular networks supporting muscle coordination, beyond muscle weakness. In fact, the correlation analyses suggested that the data of signal synergy and muscle connectivity were significantly correlated. Further, both the detrimental muscle connectivity and the signal synergy impairment were unrelated to muscle weakness. These data also substantiate the reason why to recover muscle coordination through patient-tailored rehabilitation programs may be helpful 
to improve functional gait. It will be indeed necessary not only addressing muscle weakness but also gaitrelated muscle connectivity to improve functional gait in such patients. However, larger-sample studies are necessary to confirm this statement.

Interestingly, our data suggest that muscle network impairment may depend primarily on a failure of the subtending CNS networks. At least two evidences may support this issue.

1) a disorganized muscle network pattern, in parallel to the impoverishment of available signal synergies during gait, leans more to a primary than maladaptive muscle network organization. The topological indices of CNS connectivity are reported as relatively preserved within and upstream the motor networks, thus suggesting the activation of compensatory, albeit maladaptive, mechanisms of brain plasticity to contrast muscular impairment [44]. We instead found a commingling of the patterns of muscle coordination that occur to adapt to or to recovery from brain damage (e.g., stroke), including preservation, merging, and fractionation of signal synergies [59]. Specificaaly, the abnormalities in IMCoh, PDC, and network topology suggest that motor networks are capable to generate only weak neural coupling from premotor to motor areas, with a consequential signal distortion and, eventually, muscle network fragmentation and disorganization.

2) we did not find any correlation between muscle weakness and coordination, so that neuromuscular networking impairment is likely not to depend on peripheral factors.

\section{Limitations and conclusions}

Given the small number of enrolled patients, our data are not easily generalizable, taking also into account that patients with DM1 can be significantly heterogeneous even within the same family. Thus, larger samples are required to confirm our promising data. In particular, it would be important to stratify network analysis by age and disease duration, as the progressivity of DM1 can affect gait parameters even year by year. A putative contribute of spinal network should not be neglected. In fact, IMCoh and synergy organization depends also on spinal networks and central pattern generators, which may be affected either primarily or secondarily to CNS and/or muscle impairment $[52,53]$.

One may observe that muscle connectivity data in both directed and undirected networks and signal synergies were common across patients despite their heterogeneity. Indeed, a certain degree of consistency in brain connectivity abnormalities (that we hypothesized to be reflected by signal synergies) has been shown among patients with DM1, despite their clinical heterogeneity [11]. We may postulate that the balance between the loss of connectivity within motor and premotor areas and compensatory mechanisms in some others (e.g., the cerebellum) might explain the mismatch between brain connectivity impairment and the motor and non-motor symptomatological scenario observed in these patients $[11,44]$. We may also speculate that such a conservative connectivity impairment among patients suggests more a primary trait of the disease rather that a compensative or maladaptive process to muscle dystrophy.

Anyway, our work suggests that gait and balance impairment also depend on specific CNS mechanisms that are primarily pathologic, rather than maladaptive to muscle deterioration. This information may be useful concerning the implementation of proper rehabilitative strategies in patients with DM1. It will be indeed necessary not only addressing muscle weakness but also muscle coordination during gait to improve functional gait in such patients. About that, plasticity-targeting neurorehabilitative interventions could be required. Last, the electrophysiological assessment we performed may be useful to more objectively: 1) determine the patients who may benefit from a specific intervention; 2) track gains in motor functions; 3) compare treatment strategies; and 4) tailor the treatment to the specific patient's needs.

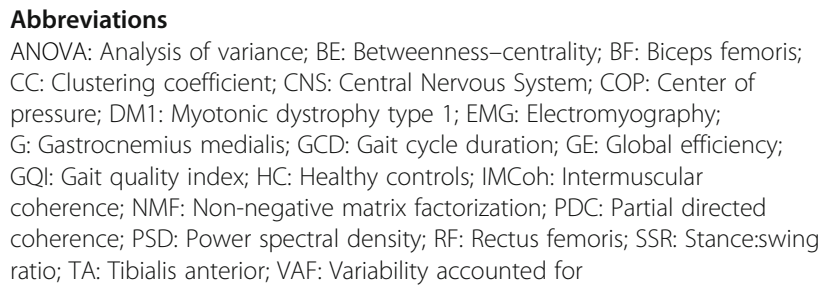

\section{Authors' contributions}

AN research project execution; statistical analysis design, execution; writing of the first draft; SP research project execution, organization; DM statistical analysis review and critique; $L B$ research project execution, organization; $A L$ research project execution; DM statistical analysis review and critique; $P B$ manuscript review and critique; RSC research project conception, organization; manuscript review and critique. All authors read and approved the final manuscript

\section{Funding}

No funding to report.

\section{Availability of data and materials}

Please contact the corresponding author for data requests.

\section{Ethics approval and consent to participate}

All procedures performed in studies involving human participants were in accordance with the ethical standards of the institutional and/or national research committee and with the 1964 Helsinki declaration and its later amendments or comparable ethical standards. The Ethics Committee of the IRCCS Centro Neurolesi Bonino Pulejo (Messina, Italy) approved the study (IRCCSME\#41/2017). 
Written informed consent was obtained from all individual participants included in the study. None of the patients experienced cognitive problems.

\section{Consent for publication}

Not applicable.

\section{Competing interests}

The authors declare that they have no competing interests.

\section{Author details}

${ }^{1}$ IRCCS Centro Neurolesi Bonino Pulejo, via Palermo, SS 113, Ctr. Casazza, 98124 Messina, Italy. ${ }^{2}$ Stomatodental Center, Messina, Italy.

\section{Received: 23 May 2019 Accepted: 9 September 2019} Published online: 18 September 2019

\section{References}

1. Udd B, Krahe R. The myotonic dystrophies: molecular, clinical, and therapeutic challenges. Lancet Neurol. 2012;11:891-905.

2. Sansone VA. The dystrophic and nondystrophic Myotonias. Continuum (Minneap Minn). 2016;22(6):1889-915.

3. Valaperta $R$, Sansone $V$, Lombardi $F$, et al. Identification and characterization of DM1 patients by a new diagnostic certified assay: neuromuscular and cardiac assessments. Biomed Res Int. 2013;2013:958510.

4. Missaoui B, Rakotovao E, Bendaya S, Mane M, Pichon B, Faucher M, Thoumie P. Posture and gait abilities in patients with myotonic dystrophy (Steinert disease). Evaluation on the short-term of a rehabilitation program. Ann Phys Rehabil Med. 2010;53(6-7):387-98

5. Pangilinan $\mathrm{PH}$, Hornyak JE. Rehabilitation of the muscular dystrophies. Handb Clin Neurol. 2013:110:471-81.

6. Hicks JE. Role of rehabilitation in the management of myopathies. Curr Opin Rheumatol. 1998;10(6):548-55.

7. Moore DP, Kowalske KJ. Neuromuscular rehabilitation and Electrodiagnosis: myopathy. Arch Phys Med Rehabi1. 2000;81:S32-5.

8. Wright RB, Yoder DM, Costa JL, Andriacchi TP. Characterization of gait parameters in adult-onset myotonic dystrophy: abnormal hip motion. Arch Phys Med Rehabil. 1995;76:33-8.

9. Galli M, Cimolin V, Crugnola V, et al. Gait pattern in myotonic dystrophy (Steinert disease): a kinematic, kinetic and EMG evaluation using 3D gait analysis. J Neurol Sci. 2012;314:83-7.

10. Tiffreau V, Detrembleur C, Van Den Bergh P, Renders A, Kinet V, Lejeune T. Gait abnormalities in type 1 myotonic muscular dystrophy: 3D motion analysis, energy cost and surface EMG. Comput Methods Biomech Biomed Engin. 2012;15(Suppl. 1:171-2.

11. Serra L, Silvestri G, Petrucci A, Basile B, Masciullo M, Makovac E, Torso M, Spanò B, Mastropasqua C, Harrison NA, Bianchi ML, Giacanelli M, Caltagirone C, Cercignani M, Bozzali M. Abnormal functional brain connectivity and personality traits in myotonic dystrophy type 1. JAMA Neurol. 2014:71(5):603-11.

12. Singh RE, lqbal $K$, White $G$, Hutchinson TE. A systematic review on muscle synergies: from building blocks of motor behavior to a neurorehabilitation tool. Appl Bionics Biomech. 2018;2018:3615368.

13. Leo A, Handjaras G, Bianchi M, Marino H, Gabiccini M, Guidi A, et al. A synergy-based hand control is encoded in human motor cortical areas. Elife. 2016;5:e13420.

14. Huffmaster SLA, Van Acker GM 3rd, Luchies CW, Cheney PD. Muscle synergies obtained from comprehensive mapping of the primary motor cortex forelimb representation using high-frequency long-duration ICMS. J Neurophysiol. 2017;118(1):455-70.

15. Di Paolo G, Jimenez-Moreno C, Nikolenko N, et al. Functional impairment in patients with myotonic dystrophy type 1 can be assessed by an ataxia rating scale (SARA). J Neurol. 2017;264(4):701-8.

16. Wojtara T, Alnajjar F, Shimoda S, Kimura H. Muscle synergy stability and human balance maintenance. J NeuroEng Rehab. 2014;11:129.

17. Boonstra TW, Danna-Dos-Santos A, Xie HB, Roerdink M, Stins JF, Breakspea M. Muscle networks: connectivity analysis of EMG activity during postural control. Sci Rep. 2015:5:17830.

18. Ghapanchizadeh H, Siti Aqlima A, Asnor Juraiza I, Maged Saleh Saeed A. Review of surface electrode placement for recording electromyography signals. Biomed Res. 2016;2017:S1-7.
19. Nishihara K, Isho T. Location of Electrodes in Surface EMG. In: Schwartz M, editor. EMG Methods for Evaluating Muscle and Nerve Function. Rijeka: Intech Open; 2012.

20. Blanc $Y$, Dimanico U. Electrode placement in surface electromyography (sEMG) "minimal crosstalk area" (MCA). OJTR. 2010;3:110-26.

21. lida $\mathrm{H}$, Yamamuro $\mathrm{T}$. Kinetic analysis of the center of gravity of the human body in normal and pathological gaits. J Biomech. 1987;20(10):987-95.

22. Kerkman JN, Daffertshofer A, Gollo LL, Breakspear M, Boonstra TW. Network structure of the human musculoskeletal system shapes neural interactions on multiple time scales. Sci Adv. 2018;4:eaat0497.

23. Sporns O, Chialvo DR, Kaiser M, Hilgetag CC. Organization, development and function of complex brain networks. Trends Cogn Sci. 2004;8:418-25.

24. Boonstra TW. The potential of corticomuscular and intermuscular coherence for research on human motor control. Front HumNeurosci. 2013;7:855.

25. Schnitzler A, Gross J. Normal and pathological oscillatory communication in the brain. Nat Rev Neurosci. 2005;6:285-96.

26. Trudeau RJ. Introduction to graph theory. Dover Pubns; 1994

27. Vecchio F, Miraglia F, Maria Rossini P. Connectome: graph theory application in functional brain network architecture. Clin Neurophysiol Pract. 2017;2:206-13.

28. Zalesky A, Fornito A, Bullmore E. On the use of correlation as a measure of network connectivity. Neuroimage. 2012;60:2096-106.

29. Watts DJ, Strogatz SH. Collective dynamics of 'small-world' networks. Nature. 1998:393:440-2

30. Rubinov M, Sporns O. Complex network measures of brain connectivity: uses and interpretations. Neuroimage. 2010;52:1059-69.

31. Merkle LA, Layne CS, Bloomberg JJ, Zhang JJ. Using factor analysis to identify neuromuscular synergies during treadmil walking. J Neurosci Methods. 1998;82:207-14.

32. Cichocki A, Zdunek R, Phan AH, Amari S. Nonnegative matrix and tensor factorizations: applications to exploratory multi-way data analysis and blind source separation. 1edition. Noida: Wiley; 2009

33. Lee $D$, Seung $S$. Algorithms for non-negative matrix factorization. Adv Neural Inf Process Syst. 2001;13:556-62.

34. Tresch MC, Cheung VCK, d'Avella A. Matrix factorization algorithms for the identification of muscle synergies: evaluation on simulated and experimental data sets. J Neurophysiol. 2006;95(4):2199-212.

35. Vasco G, Petrarca M, D'Amico A, Colia G, Catteruccia M, Gazzellini S, Carniel S, Pisano A, Castelli E, Bertini E. Functional and gait assessment in children with Duchenne muscular dystrophy: quantitative and functional evaluation. Neuromuscul Disord. 2015:25(S2):230-1.

36. Tiffreau $V$, Detrembleur $C$, Van Den Bergh $P$, Renders A, Kinet $V$, Thevenon A, Allart E, Lejeune T. Gait among patients with myotonic dystrophy type 1: a three-dimensional motion analysis study. J Int Soc Phys Rehabil Med. 2018;1:65-71.

37. Trias D, Gioux M, Cid M, Bensch C. Gait analysis of myopathic children in relation to impairment level and energy cost. J Electromyogr Kinesiol. 1994; 4(2):67-81.

38. Goudriaan M, Shuman BR, Steele KM, et al. Non-neural muscle weakness has limited influence on complexity of motor control during gait. Front Hum Neurosci. 2018;12:5.

39. de León MB, Cisneros B. Myotonic dystrophy 1 in the nervous system: from the clinic to molecular mechanisms. J Neurosci Res. 2008;86(1):18-26.

40. Johnson A. Myotonic dystrophy. In: Lalwani K, Todd-Cohen I, Choi EY, Raman VT, editors. Pediatric Anesthesia: A Problem-Based Learning Approach. New York: Oxford University Press; 2018. p. 315-21.

41. Bosco G, Diamanti S, Meola G, DM-CNS Group. Workshop report: consensus on biomarkers of cerebral involvement in myotonic dystrophy, 2-3 December 2014, Milan, Italy. Neuromuscul Disord. 2015;25(10):813-23.

42. Spedden ME, Jensen P, Terkildsen CU, Jensen NJ, Halliday DM, LundbyeJensen J, Nielsen JB, Sparre Geertsen S. The development of functional and directed corticomuscular connectivity during tonic ankle muscle contraction across childhood and adolescence. Neurolmage. 2019;191:350-60.

43. Buzsaki G, Draguhn A. Neuronal oscillations in cortical networks. Science. 2004:304:1926-9.

44. Serra L, Mancini M, Silvestri G, et al. Brain Connectomics' modification to clarify motor and nonmotor features of myotonic dystrophy type 1. Neural Plast. 2016;2016:2696085

45. van Dorst M, Okkersen K, Kessels RPC, Meijer FJA, Monckton DG, van Engelen BGM, Tuladhar AM, Raaphorst J. OPTIMISTIC consortium. Structural white matter networks in myotonic dystrophy type 1. Neuroimage Clin. 2019;21:101615 
46. Minnerop M, Gliem C, Kornblum C. Current Progress in CNS imaging of myotonic dystrophy. Front Neurol. 2018;9:646.

47. Rana M, Yani MS, Asavasopon S, Fisher BE, Kutch JJ. Brain connectivity associated with muscle synergies in humans. J Neurosci. 2015;35(44): 14708-16.

48. Yokoyama H, Kaneko N, Ogawa T, Kawashima N, Watanabe K, Nakazawa K. Cortical control of locomotor muscle activity through muscle synergies in humans: a neural decoding study. iscience. 2019;15:623-39.

49. Ting LH, McKay JL. Neuromechanics of muscle synergies for posture and movement. Curr Opin Neurobiol. 2007;17(6):622-8.

50. Boonstra TW, Breakspear M. Neural mechanisms of intermuscular coherence: implications for the rectification of surface electromyography. J Neurophysiol. 2012;107:96-807.

51. Farina D, Negro F, Jiang N. Identification of common synaptic inputs to motor neurons from the rectified electromyogram. J Physiol. 2013;591: 2403-18.

52. Hart CB, Giszter SF. A neural basis for motor primitives in the spinal cord. J Neurosci. 2010;30:1322-36.

53. Allum JH, Bloem BR, Carpenter MG, Hulliger M, Hadders-Algra M. Proprioceptive control of posture: a review of new concepts. Gait Posture. 1998;8:214-42

54. Fierro B, Daniele O, Aloisio A, Buffa D, La Bua V, Oliveri M, Manfre L, Brighina F. Neurophysiological and radiological findings in myotonic dystrophy patients. Eur J Neurol. 1998:5(1):89-94.

55. Oliveri M, Brighina F, La Bua V, Aloisio A, Buffa D, Fierro B. Magnetic stimulation study in patients with myotonic dystrophy. Electroencephalogr Clin Neurophysiol. 1997;105(4):297-301.

56. Portaro S, Naro A, Chillura A, Billeri L, Bramanti A, Bramanti P, Rodolico C, Calabrò RS. Toward a more personalized motor function rehabilitation in myotonic dystrophy type 1: the role of neuroplasticity. PLoS One. 2017; 12(5):e0178470.

57. Heitmann S, Boonstra TW, Gong P, Breakspear M, Ermentrout B. The rhythms of steady posture: motor commands as spatially organized oscillation patterns. Neurocomp. 2015;170:3-14.

58. García-Cossio E, Broetz D, Birbaumer N, Ramos-Murguialday A. Cortex integrity relevance in muscle synergies in severe chronic stroke. Front Hum Neurosci. 2014:8:744

59. Cheung VC, Turolla A, Agostini M, Silvoni S, Bennis C, Kasi P, Paganoni S, Bonato $\mathrm{P}$, Bizzi E. Muscle synergy patterns as physiological markers of motor cortical damage. Proc Natl Acad Sci U S A. 2012;109(36):14652-6.

\section{Publisher's Note}

Springer Nature remains neutral with regard to jurisdictional claims in published maps and institutional affiliations.

Ready to submit your research? Choose BMC and benefit from:

- fast, convenient online submission

- thorough peer review by experienced researchers in your field

- rapid publication on acceptance

- support for research data, including large and complex data types

- gold Open Access which fosters wider collaboration and increased citations

- maximum visibility for your research: over $100 \mathrm{M}$ website views per year

At BMC, research is always in progress.

Learn more biomedcentral.com/submissions 